LAW RENCE LIVERMORE N A T IO N A L LABORATORY
Response to Questions on
Presentation to NAS

W. R. Meier

March 18, 2011 
This document was prepared as an account of work sponsored by an agency of the United States government. Neither the United States government nor Lawrence Livermore National Security, LLC, nor any of their employees makes any warranty, expressed or implied, or assumes any legal liability or responsibility for the accuracy, completeness, or usefulness of any information, apparatus, product, or process disclosed, or represents that its use would not infringe privately owned rights. Reference herein to any specific commercial product, process, or service by trade name, trademark, manufacturer, or otherwise does not necessarily constitute or imply its endorsement, recommendation, or favoring by the United States government or Lawrence Livermore National Security, LLC. The views and opinions of authors expressed herein do not necessarily state or reflect those of the United States government or Lawrence Livermore National Security, LLC, and shall not be used for advertising or product endorsement purposes.

This work performed under the auspices of the U.S. Department of Energy by Lawrence Livermore National Laboratory under Contract DE-AC52-07NA27344. 


\title{
Response to Questions on Presentation to NAS*
}

\author{
Wayne R. Meier \\ Lawrence Livermore National Laboratory
}

March 16, 2011

*Response to questions on the presentation “Overview to Chamber and Power Plant Designs for IFE” made at the 1/29-31 meeting of the National Academies' Committee on the Prospects for Inertial Confinement Fusion Energy Systems.

This work was performed under the auspices of the U.S. Department of Energy by Lawrence Livermore National Laboratory under Contract DE-AC52-07NA27344. 


\section{Questions for Wayne Meier}

Question (F1): It would be helpful to policy-makers to have a side-by-side comparison of the key scientific issues and parameters important for the national security objective "versus" the commercial energy performance objective. Please provide your perspective on this.

\section{Answer:}

This is a high level question that is more suitably answered by Laboratory Senior Management. I respectfully defer to others who have also been asked this question.

Question (B1): As I understand it, the idea of having an atmosphere of 0.5 Torr of Xe to protect the first wall was incompatible with a cryogenic target because of "heating" of the target. Naturally, Xe would be condensing on the target, contributing an enormous heat load.

But the target could be housed in a very thin aluminum vacuum thimble, arranged so that it could be flicked off just a few milliseconds before shot time. In fact, the vacuum thimble could be double-walled, so that the target (or the target within the hohlraum) could be in a $0.5 \mathrm{~T}$ hydrogen atmosphere, so that when the thimble is flicked off, the He will not rush into the vacuum but would only gradually replace the $\mathrm{H} 2$ as the latter began to rise by buoyancy in the Xe atmosphere. A simple calculation shows that the buoyancy flow is irrelevant for a time of, for instance, $0.01 \mathrm{~s}$, because as is well known a bubble in gas rises with an acceleration of $2 \mathrm{~g}$, so that the amount of rise would be about $0.3 \mathrm{~cm}$ in $0.01 \mathrm{~s}$..

It is hard to believe that this was not proposed before, but it seems a shame to give up the 0.5-T Xe protection simply because of the heating that could, it seems, be readily countered in this way.

\section{Answer:}

There have been several conceptual ideas proposed for protecting a direct drive target while in transit through the hot chamber. These ideas are at a very preliminary level and have not received the level of rigorous analyses and experimental testing needed to show feasibility. The paper by Shmatov et al. [1] deals with this subject as described in the abstract:

Protection of the direct-drive thermonuclear targets in the reaction chamber by containers of several kinds is considered. Thermoinsulation of the cryogenic thermonuclear fuel by a target ablator consisting of two layers separated by vacuum, low-density gas, or low-density foam is also considered. It is shown that the cones that provide a beam channel for heating the compressed fuel in some of the fast ignition, direct compression scenarios can also serve as effective wake shields protecting the fuel capsules.

The paper provides top level analyses of some of the ideas including something similar to the "thermos bottle" idea mentioned in this question. A copy of the paper has been forward to NAS.

[1] M. L. Shmatov, R.W. Petzoldt, E.I. Valmianski, "Measures to Provide Survival of the Direct Drive and Fast Ignition, Direct Compression Targets in the Reaction Chamber," Fusion Science and Technology, 43, 312 (2003). 
Question (B2): Some of the approaches to fusion are amenable to heating-systems primarily on the axis of the target. These could protect the first wall by the use of a thick liquid blanket, as was shown in several of the slides. There is a simple way of shielding a cylindrical first wall of whatever diameter and with a liquid of whatever thickness. For instance, take a first wall at a distance of $3 \mathrm{~m}$, and cover its inside with 1-m-thick annulus of FLiBe or some other suitable material. If the axis of the system is horizontal, one could make the first wall perhaps 10-m long, and set it into rotation about the horizontal axis. If it is going fast enough so that the centripetal acceleration is several $g$, the liquid is stationary in the rotating cylinder. Of course, there will need to be end caps to contain the liquid, but the end caps need not project farther toward the axis than the liquid itself. Dissipation in the liquid, by residual flow under gravity could be minimized by fins in the centrifuge.

Such a system is often used for "centrifugal casting" of cast-iron or steel water pipe or other materials. Of course, one needs to arrange a flow of the protection/heat-transfer fluid, which is also the blanket, and for that one needs to have rotating seals, preferably on the axis. If necessary, toroidal joints could be used.

There are many options for bearings, because this service is not particularly onerous.

Of course, the vacuum-chamber or gas-type chamber need not rotate. It could simply be a stationary housing in which the rotating "first wall" exists, with its inner protective blanket.

This would be most suitable for heavy ion fusion or, perhaps, for polar-drive laser fusion. I would like to know of any analyses of this approach.

Too often, there is an analysis of something that at first glance seems to be what is proposed, but it turns out to be different, so I would like to see it.

\section{Answer:}

Yes, the idea of a rotating chamber filled with a thick liquid layer was proposed by Bob Bourque (with General Atomics at that time) as part of the "new ideas" phase of the study that eventually lead to the Sombrero and Osiris designs and report [2]. This chamber design was only developed to a very preliminary conceptual level; it was not evaluated in the same detail as other more comprehensive integrated design studies. Bourque's concept, which did include a scheme for protecting the ends of the chamber, was documented in Appendix B of Ref. 2 and published in the proceedings of an IEEE SOFE meeting [3]. Ironically he dubbed the concept Liner Inertial Fusion Energy (LIFE). A copy of the paper has been forwarded to NAS.

[2] W.R. Meier, et al., “OSIRIS and SOMBRERO Inertial Confinement Fusion Power Plant Designs,” DOE/ER/54100-1 (1992).

[3] R. Bourque, "ICF Reactor Chambers with Rotating Liquid Blankets," Proc. $14^{\text {th }}$ IEEE/NPSS Symposium on Fusion Engineering (9/30 - 10/3/91), ISBN 0-7803-0132-3.

(E1) Where can we find information on the dynamics of the gas in the chamber?

\section{Answer:}

Most of the early time ( $<1 \mathrm{~ms}$ ) modeling of chamber gas response has been carried out by University of Wisconsin staff using the BUCKY code [e.g., 4]. There has not been a lot published on the longer time evolution of chamber conditions, but some work was done by 
UCSD for the HAPL program [5-7]. The LIFE team is currently modeling various aspects of the LIFE chamber dynamics and is planning experiments to similute the post-shot environment. The point of contact for this and other LIFE related work is Mike Dunne.

[4] R.R Peterson et al., "Inertial fusion energy target output and chamber response:

Calculations and experiments,” Physics of Plasmas, 9, No. 5, 2287 (2002).

[5] Z. Dragojlovic, F. Najmabadi, "Effects of chamber geometry and gas properties on hydrodynamic Evolution of IFE chambers,” Fusion Science and Technology, 47, 1152 (2005).

[6] Z. Dragojlovic, F. Najmabadi, "Simulation of IFE chamber dynamic response by a second order Godunov method with arbitrary geometry,” Proceedings of Third International Conference on Inertial Fusion Science and Applications, (American Nuclear Society), Monterey, California, September 7-12, 2003, pp. 850-853.

[7] HAPL presentations:

F. Najmabadi and Z Dragojlovic, "Progress Report on SPARTAN Simulation of IFE Chamber Dynamics,” (March 3-4, 2005). http://aries.ucsd.edu/HAPL/MEETINGS/0503-HAPL/najmabadi-spartan.pdf

Z. Dragojlovic and F. Najmabadi, "SPARTAN Chamber Dynamics code” (June 20-21, 2005). http://aries.ucsd.edu/HAPL/MEETINGS/0506-HAPL/program.html

Question (G33): Can we get PDFs of the economic studies listed in the slides? (G26) Such as, "McCarville T.J., Meier* W.R., Carson* C.R., and Glasgow* B. SAFIRE-A Systems Analysis Code for ICF Reactor Economics. 1987." Also, is that code available, still?

\section{Answer:}

Yes, PDFs have been forwarded to NAS and will be placed in a repository made available to the committee.

No, the SAFIRE code is no longer in use or available, but the algorithms are documented in the SAFIRE report. There are also simple economic scaling algorithms for the Sombrero and Osiris power plants documented in Refs. 8 and 9, but readers are cautioned that these are 20 years old. The most up to date economic studies are being completed by the LIFE team.

[8] W.R. Meier, R. L. Bieri, "Economic Modeling and Parametric Studies for Osiris - An HIF-Driven IFE Power Plant,” Fusion Technology, 21, 1547 (1992).

[9] W.R. Meier, C.W. vonRosenberg, Jr., "Economic Modeling and Parametric Studies for Sombrero - A Laser-Driven IFE Power Plant,” Fusion Technol., 21, 1552 (1992).

Question (F4): What are the relative potential advantages and disadvantages of direct drive and indirect drive, generally?

\section{Answer:}

This is a very broad question that could be addressed from a variety of viewpoints (e.g., target performance, target physics theoretical and experimental basis, target fabrication and injection, driver/ target interface, chambers, etc.). In fact the question would best be addressed by 
comparing the complete integrated designs of power plants based on direct and indirect drive targets. Clearly there are too many combinations of drivers, chamber and targets to provide generic answers to the question. Since my talk to NAS focused on chamber designs, I will limit my comments to the relative advantages and disadvantages from the perspective of the chamber designer. From that perspective, there are two key considerations: 1) allowing for drive energy delivery (e.g., beam pathways) and 2) how the chamber deals with target emissions.

Dry-wall chambers easily accommodate the illumination geometry for either direct or indirect drive. For laser drivers, chamber designs have be proposed to deal with target emission from either direct (e.g., HAPL) or indirect drive (e.g., LIFE) targets. As discussed in the presentation, each has its own issues to resolve. Direct drive targets have a higher fraction of high energy ions, which requires chamber design measures (e.g., magnetic diversion) to prevent ion implantation in the first wall. The emission spectrum from indirect drive targets can be stopped in a chamber fill gas, which simplifies the chamber structural design. To my knowledge, there are no detailed studies of dry-wall concepts for either direct or indirect dry with heavy ion or Z pinch drivers.

Wetted wall chambers could be compatible with either direct or indirect drive illumination, but there is some advantages to indirect drive since it would possible to configure the beams path from the sides and this could reduce the chance of liquid reaching final optics. The thin liquid layer would be able to handle short range emission from either direct or indirect drive targets, so there is little difference there.

Thick liquid walls are likely only compatible with indirect drive targets unless extraordinary measures are taken in an attempt to provide a thick shielding region between up to hundreds of beam paths. The thick liquid layer easily handles the target emissions. Indirect drive with thick liquid wall chambers is the primary choice of both heavy ion and Z-pinch driver.

Question (G30): What possibilities are there with your drivers for D-HE3 or P-boron fusion?

\section{Answer:}

I don't have the expertise in target physics to answer this question, but there have been many published studies dating back to the mid 1970's. John Perkins (LLNL) would be a good contact for literature on this topic.

Question (I1): What is the significance of driver efficiency for power plant credibility?

\section{Answer:}

Driver efficiency is more of an attractiveness issue than credibility issues, at least for types of drivers currently being considered for IFE (i.e., all >7\%). The driver efficiency impacts the capital cost and cost of electricity (COE) of the plant. Increasing driver efficiency reduces the driver recirculating power fraction and reduces the COE if everything else is held constant; there is simply more net power to sell. But there are trade-offs that must be considered. When we hold the net power of the plant fixed, we find that the cost of electricity is relatively insensitive to efficiency. As efficiency decreases, the driver design typically moves to somewhat larger driver energy resulting in a higher target gain which lowers the recirculating power. The capital cost of 
the plant scales less than linearly with gross power, so there is an economy of scale for the larger plant with more gross electric power. Also for a given driver, higher efficiency typically comes at a cost, and this needs to be included in the systems analyses of the trade-offs.

In summary driver efficiency is an important factor affecting the economic attractiveness of the plant, but flexibility in selecting the plant operating space tends to reduce its impact on overall economics. Designs tend to reach a point of diminishing returns in pushing up driver efficiency and/or driver energy for higher target gain. That is, above a certain $\eta \mathrm{G}$ product, there are diminishing economic returns.

Question (G31): What spin off possibilities do you see from your R\&D?

\section{Answer:}

Spins offs are difficult to predict. In the area of chamber R\&D there certainly could be spin-offs from the material science being carried out for first walls, both in terms of high heat flux capabilities and radiation damage resistance. Another area that is already seeing some spin offs is experimental and modeling work related to pulse heating and ablation modeling. UCSD is applying their expertise to other applications such as ion sources for accelerators and producing plasma for EUV x-ray sources for various applications [10].

[10] R. A. Burdt et al., Recombination effects during expansion into vacuum in laser produced Sn plasma," Applied Physics Letters, 97, 041502 (2010). 